\title{
How to Modulate the Immunoinflammatory Biological Response in Cancer, Autoimmunity and Viral Infections by a Psychoneuroimmune Approach
}

ISSN: 2637-773X

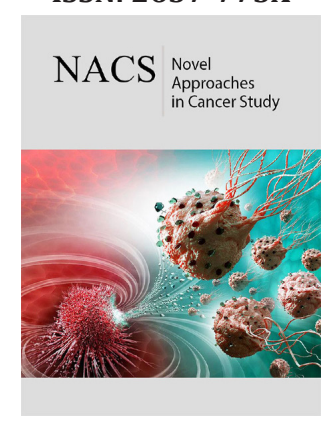

*Corresponding author: Paolo Lissoni, Institute of Biological Medicine, Milan, Italy

Submission: 眥 January 18, 2021

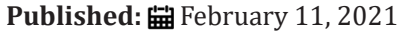

Volume 5 - Issue 5

How to cite this article: Paolo Lissoni, Fernando Brivio, Luca Fumagalli, Franco Rovelli, Massimo Colciago, et al. How to Modulate the Immunoinflammatory Biological Response in Cancer, Autoimmunity and Viral Infections by a Psychoneuroimmune Approach. Nov Appro in Can Study. 5(5). NACS.000622. 2021. DOI: 10.31031/NACS.2021.05.000622

Copyright@ Paolo Lissoni, This article is distributed under the terms of the Creative Commons Attribution 4.0 International License, which permits unrestricted use and redistribution provided that the original author and source are credited.
Paolo Lissoni ${ }^{1 *}$, Fernando Brivio ${ }^{1}$, Luca Fumagalli ${ }^{2}$, Franco Rovelli ${ }^{1}$, Massimo Colciago $^{3}$, Giusy Messina ${ }^{1}$, Alejandra Monzon ${ }^{1}$, Francesco Pelizzoni ${ }^{1}$, Arianna Lissoni $^{1}$, Giorgio Porro ${ }^{1}$, Giuseppe Di Fede ${ }^{1}$ and Daniel P Cardinali ${ }^{4}$

${ }^{1}$ Institute of Biological Medicine, Italy

${ }^{2}$ Surgical Division, Manzoni Hospiltal, Lecco, Italy

${ }^{3}$ INRCA-IRCCS Institute, Casatenovo, Lecco, Italy

${ }^{4}$ Facultad de Ciencias Médicas, Pontificia Universidad Católica Argentina,Buenos Aires, Argentina

\begin{abstract}
Recent advances in the area of the psycho-neuroendocrine-immunology have demonstrated that the inflammatory and immune reactions depend not only on immune factors, but also, they are under an intricate neuroendocrine regulation. In turn, the activity of the nervous and endocrine systems is affected by immune-inflammatory signals. Although most hormones and neuromodulators can potentially influence the immune function, main immunomodulatory properties seem to be exerted by opioid and cannabinoid systems. Brain opioid and cannabinoid systems are endocrinologically connected to the adrenal, and to the pineal gland, respectively. In addition, the neurohypophysis is also important for the neuroimmunomodulation through the release of vasopressin and oxytocin. Experimental evidence supports the conclusion that two fundamental functional circuits occur, including a brain opioid system-vasopressin-adrenal axis, characterized by a prevalent immunosuppressive activity, and a brain cannabinoid-oxytocin-pineal axis, which exerts an immunostimulatory role. The latter is relevant mainly for anticancer immunity. The opioid-adrenal functional axis is active in stress and depressive conditions, while the cannabinoid-pineal axis has been proven to be involved in pleasure perception and spiritual expansion of mind. The findings could explain the predisposition to severe systemic diseases played by stress and depression, and on the other hand the protective action of pleasure and spirituality in the onset of important pathologies. The clinical and therapeutic applications of these concepts may constitute a landmark for future Medicine.
\end{abstract}

Keywords: Autoimmunity; Biological response; Cancer; Cannabinoids; Covid-19; Cytokines; Melatonin; Neuroimmunomodulation

\section{Introduction}

It is a biological evidence that the structure of the immune system reflects the whole history of life in our Planet, T lymphocytes being the most evolved cells of the human immune system. In fact, despite the great complexity of the immune system, the foundations of its function are substantially based on three main $\mathrm{T}$ lymphocyte subsets, namely $\mathrm{T}$ helper lymphocytes (Th) (CD4+), regulatory T lymphocytes (T reg) (CD4+CD25+) and T17 lymphocytes (Th17) $(\mathrm{CD} 4+\mathrm{CD} 17+)$. Like a trinity, they underlie the unity of the whole immune system, and of overall immune functions through their connections with other less evolved immune cells. Communication among the different immune cells is conveyed through the release of a great number of proteins, namely cytokines and chemokines. Some cytokines are called interleukins (IL). While the chemokines mainly act at local level, the cytokines tend to exert systemic effects, not only on the immune system, but also on the overall biological systems, including the endocrine system, the nervous system and the cardiovascular apparatus [1]. Then, the cytokines may be considered as the equivalent of the hormones for the endocrine system. Th17 cells are mainly linked to the macrophage system for the activation of the inflammatory response, as well as they suppress the function of T reg lymphocytes [2]. The lymphocytes, whose most important cytokine is IL-2 [3], are mainly connected with the dendritic cells, 
and their generation is promoted by the dendritic cells through the release of IL-12 [4], which also activate the antigen-dependent cytotoxicity of cytotoxic T lymphocytes (CD8+). Th cells activate $\mathrm{T}$ reg lymphocytes [5], inhibit the Th17 cells [6], and promote the antigen-independent cytotoxicity by inducing the evolution of natural killer (NK) cells into LAK cells [3]. The most important actions of Th cells are accomplished via the secretion of IL-2, the main growth factor for T lymphocytes [3]. Moreover, IL-2 is the only cytokine able to in vivo enhance the number of lymphocytes [7].Then, Th1lymphocytes are the only immune cells with self-stimulation capability by releasing IL-2 and expressing the receptor for IL-2 (IL2R) [3]. The lymphocytes exist into two different forms, Th1 and Th2, which is probably a functional distinction and not two different populations of cells, since at present no cluster of differentiation has been identified to distinguish between Th1 and Th2 cells. Naïve Th cells can be differentiated into Th1 cells under the stimulation of IL-12 released from the dendritic cells, or into Th2 cells by IL4, mainly released from mast cells and basophils [4]. Th2 cells are fundamental for the activation of B lymphocytes (CD19+CD20+), and their evolution into plasma cells, which are the source of all circulating antibodies. Additionally, T reg lymphocytes control the inflammatory response by the release of the anti-inflammatory cytokines IL-10 and TGF-beta [8]. The function of the immune cells is and so influenced by metabolic agents, such as vitamin D, hormones, and mainly by neurohormones, neurotransmitters, and neuromodulators, which represent the mechanisms responsible for the mediation of the influence of the psychological and the spiritual life on the biological body, by regulating the immune system and its reactivity to the different stimulations. All immune cells are potentially influenced by the different hormonal and neuroactive agents, but the most important neuroendocrine regulation is that regarding the three major immune cells, corresponding to the three fundamental T cell subsets.

\section{Relationships Among Th1, T RegAnd Th17 Lymphocytes}

The relationships occurring among the three major $\mathrm{T}$ lymphocyte subsets constitute fundamental biomarkers of main human systemic diseases, including cancer, autoimmune pathologies, and infectious diseases. Three relevant ratios include Th1-to-T reg cell ratio (Th1/T reg), Th17-to-T reg cell ratio (Th17/T reg), and Th1-to-Th17 cell ratio (Th1/Th17). Since CD4+ count is ranging from $35 \%$ to $55 \%$ of total lymphocytes, $\mathrm{T}$ reg cells range from $5 \%$ to $10 \%$ of total CD $4+$ cells, and Th17 cells from $3 \%$ to $8 \%$ of total CD $4+$ cells, the normal values for the three ratios are: Th1/T reg ratio $>10$, Th17/T reg ratio $<0.8$, and Th1/Th17 ratio $>15$. An abnormally low Th1/T reg ratio is the main characteristic of the advanced neoplasms, depending on a decrease in Th1 cell count in association with an increase in T reg cell number [9]. An increase in Th17/T reg ratio, due to an increase in Th17 cells and a decline in T reg cells inhibited by the action of Th17cells [2], is the main characteristic of the autoimmune diseases. Such an increase in in Th17/T reg ratio occurs also in coronavirus-induced acute respiratory distress syndrome (ARDS), or severe acute respiratory syndrome (SARS) [10], while it is variable in the advanced neoplasms, characterized by an increase in both Th17 [11] and Treg cell counts [12]. Finally, a decreased Th1/Th17 cell ratio has been observed either in the advanced cancer, because of the concomitant occurrence of low values of Th1 cells and high values of Th17 cells, or in the autoimmune diseases, because of the greater increase in Th17 cell count with respect to Th1 cell number [13]. Additionally, the three main subsets of CD4+ cells are connected among them by complex negative and positive feedback mechanisms, as illustrated in Figure 1. In more detail, IL-2 released from Th1 cells stimulates $\mathrm{T}$ reg cell activity [5], while TGF-beta and IL-10 produced by T reg cells inhibit IL-2 secretion [8], giving rise to a negative feedback circuit. In addition, IL-2 inhibits Th17 cell function (6), while IL17 released from Th17 cells could enhance IL- 2 secretion via the inhibition of TGF-beta secretion [14], constituting another negative feedback mechanism, although it remains to be established a direct effect of IL-17 on Th1 cells and their release of IL-2. More complex is the relation between Th17 and T reg cells. In fact, while the effect of Th17 on T reg cells is constantly in an inhibitory way, the influence of $\mathrm{T}$ reg cells on Th17 cells through the release of TGF-beta is depending on the concentrations of other cytokines, namely IL-6, IL-1 beta and IL-23. TGF-beta alone may inhibit Th17 cell-related IL-17 secretion, while in the presence of high concentrations of IL6, IL-1 beta and IL-23, TGF-beta may promote Th17 differentiation and activation [15].

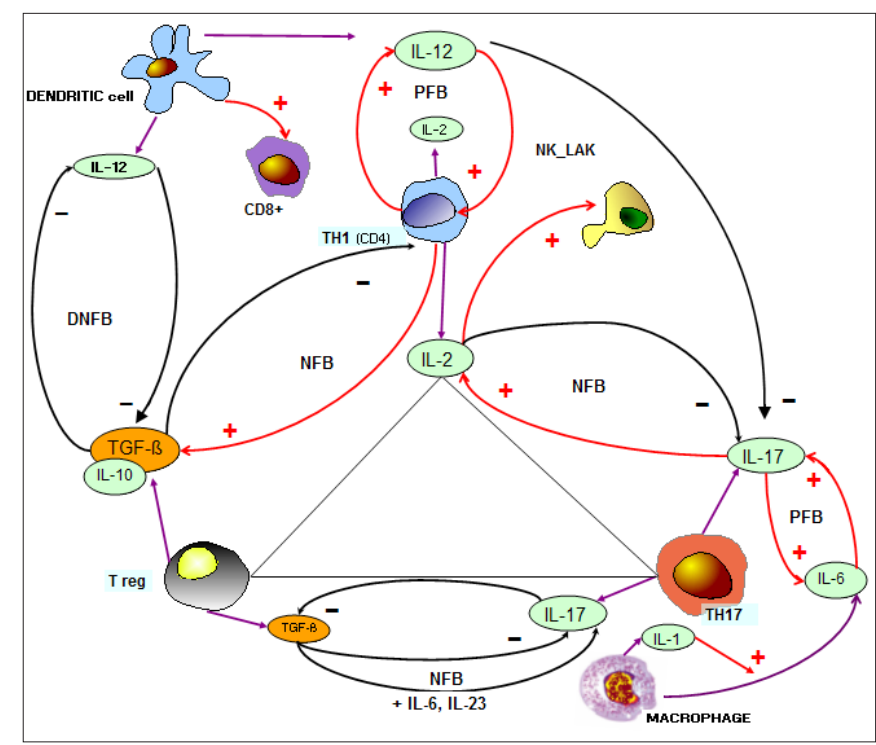

Figure 1: The trinity of Tcells and their feed-back (FB) mechanism.

NFB: Negative FB; PFB: Positive FB; DNF: Soluble Negative FB.

Therefore, either a double negative circuit, or a double negative feedback mechanism may occur between TGF-beta and IL-17 secretion, since in the presence of TGF-alone, there is a reciprocal inhibitory action between TGF-beta and IL-17, while in the presence of TGF-beta plus IL-6, there is a classical negative 
feedback mechanism, with inhibitory effects of IL-17 on T reg cells, and a stimulatory action of TGF-beta on IL-17.The three main CD4+ $\mathrm{T}$ cells are under the regulation of dendritic cells, macrophages, and endothelial cells, through the release of IL-12, IL-6 and IL-18. IL12 stimulates Th1 differentiation and IL-2 secretion [4], whereas it may inhibit both T reg [16] and Th17 lymphocytes [17]. In humans, IL-12 and IL-2 are connected by a positive feedback mechanism, since IL-2, whose secretion is stimulated by IL-12, also promotes IL-12 release from the dendritic cells [18]. The macrophage production of IL-6, which is induced by IL-1 beta released from macrophages themselves, could counteract IL-2 activity, that, in turn, may activate the macrophage system to release IL -6 [19]. T reg cells and macrophages are presumably connected by a double negative circuit, since TGF-beta released from $\mathrm{T}$ reg cells plays its anti-inflammatory action by inhibiting the pro-inflammatory effect of macrophages, while IL- 6 and IL- 1 beta produced by macrophages could counteract $\mathrm{T}$ reg cell function [8]. Finally, IL-18 is one of the most controversial cytokines because of the complexity of its effects. It is mainly produced by macrophages and endothelial cells. It may stimulate interferon (IFN) gamma production from Th1 cells in association with IL-12, and it may also play an angiogenic activity by stimulating vascular endothelial growth factor (VEGF) secretion [20]. IL-18 seems to be linked to IL-2 by a reciprocal stimulatory circuit [21]. Then, in association with macrophage- and Th17-dependent inflammatory response, IL-18 may constitute a source for the inflammatory process, by potentially amplifying both macrophage- and Th17- dependent inflammation.

\section{The Cytokine Secretion and the Physiopathology of Systemic Diseases}

By now, it is known that the symptomatology of systemic human diseases, including cancer and autoimmune pathologies, is depending on an exaggerated secretion of inflammatory cytokines, thus constituting the so-called cytokine release syndrome. The popular definition of cytokine storm is not correct, since a storm implies a similar activity of all its components, while in the cytokine release syndrome several cytokines with different functions are differentially involved. Moreover, some cytokines are abnormally released in both cancer and autoimmunity, while different profiles have been described for other cytokines. The metastatic cancer, the autoimmune diseases during their exacerbation phases, and some viral infections, namely Ebola [22] and coronaviruses [23], are all characterized by an abnormally high endogenous release of IL-6, TNF-alpha, IL-1 beta and IL-17, while some difference occur namely in the case of TGF-beta, whose secretion is abnormally enhanced in the metastatic cancer [12] and low in the acute phases of the autoimmune diseases [24]. Then, the increased levels of TGF-beta in the advanced cancer, and of diminished TGF-beta concentrations in the autoimmune pathologies are of negative prognostic significance. In addition, low levels of IL-2 together with lymphocytopenia predict a negative outcome in the advanced tumours [25]. The role of IL-2 secretion in autoimmune diseases and coronavirus infections needs to be better investigated and clarified. Similar considerations can be made concerning the profile of IL-12. In any case, must be noted that a single sampling is not enough to define the profile of cytokine secretion in the human systemic diseases, and this finding could explain the controversial results often reported in the literature concerning cytokines.

\section{The Psychoneuroendocrine Regulation of the Biological Response}

Almost all experimental and clinical investigations in the area of the psycho-neuroendocrine immunology (PNEI) have been limited to the study of the influence of stress, rather than that of the pleasure, and to the evaluation of the influence of emotions, rather than of the states of consciousness. Although most hormones and neuroactive molecules can potentially influence the inflammatory and immune responses, from a pathological point of view, five major structures have emerged as playing a fundamental role in mediating the influence of the psychospiritual status on the immune system. They include the pineal gland through its most investigated hormone melatonin (MLT) [26], the adrenal gland via the secretion of cortisol, the brain opioid system, mainly through the mu-receptor, the brain cannabinoid system, and the alpha- and beta- adrenergic system. Pineal gland and adrenal gland have opposite effects on the immune function including stimulatory and suppressive effects, respectively. Pineal and adrenal glands are also opposite in their circadian rhythmicity, MLT being mainly produced at the night, and cortisol during the early phases of the day. In addition, MLT and cortisol play opposite effects on platelet functions, since MLT stimulates platelet production [27], while cortisol reduces platelet count. As far as their effects on the inflammatory response, both pineal and adrenal glands exert anti-inflammatory effects, although via profoundly different mechanisms. On the same way, brain opioid and cannabinoid systems exert opposite effects on the immune responses, even though they are connected by several complex interactions. Finally, the influence of the sympathetic system on the immune functions depends on the different types of adrenergic receptors involved, including alpha-1, alpha-2, and beta-receptors.

MLT secretion is stimulated by noradrenaline released by neural fibers coming from the superior cervical ganglion by acting on a beta-receptor [26]. In this pathway, noradrenaline release is maximal at night, then MLT is mainly released during the dark period of the day. In addition, MLT secretion is also influenced by several other factors, since it is simulated by cannabinoids [28], while the alpha- 2 agonists play an inhibitory action. More complex and controversial are the effects of opioids on MLT secretion, since both inhibitory and stimulatory actions have been reported, depending on the different experimental conditions, dose, and the type of opioid agonists. The activity of the pineal gland is mainly connected to that of brain cannabinoid system [28,29], while the brain opioid system is more linked to the activity of the adrenal gland. Therefore, it is possible to identify two fundamental neuroendocrine functional axes, represented by pineal-cannabinoid axis, and opioid-adrenal axis. The adrenal-opioid axis is active in 
stress and depressive mood [30], while the pineal-cannabinoid one is active in the pleasure perception and expansion of consciousness $[28,29]$. In addition, the neurohypophysis is also involved in neuroimmunomodulation through the release of vasopressin and oxytocin [31], which are respectively connected to the opioidadrenal axis, and to the pineal-cannabinoid axis. As a hypothesis, the opioid-vasopressin-adrenal axis is involved in the unconscious life, such as conditions of stress, anxiety, depression, and pain, and entails immunosuppression, while the pineal-oxytocincannabinoid axis is responsible for pleasure perception, affective life, and expansion of mind, leading to immunostimulation. These mechanisms would explain how immune function is suppressed by stress and depression, and how it is augmented by pleasure, mind expansion, and love. Obviously, the biological reality is much more complex than this mechanistic description, but we need to simplify the psychobiological processes before understanding the whole systemic function, according to Descartes's principles. Finally, as far as the influence of the adrenergic system, alpha- 2 agonists have appeared to allow a condition of immunosuppression, while alpha-1 adrenergic agonists stimulate the immune system [32].

The effects of adrenergic agonists and antagonists presumably change in relation to the various immune cell subsets, and in particular it has been shown that the beta-adrenergic agonists inhibit Th1 cells, and in more intense manner the cytotoxic T lymphocytes [32], while they would play a stimulatory action on the T reg cells [33]. The main targets for MLT immunostimulatory action have been proven to be Th1 lymphocytes and dendritic cells, with a subsequent stimulation of both IL-2 and IL-12 production [34]. MLT also inhibits the macrophage production of TNF-alpha and IL-6. Lastly, MLT would exert a predominant inhibitory action on T reg [35] and Th17 lymphocytes [36], with subsequent inhibition of IL-17 and TGF-beta secretion, depending on the different clinical or experimental conditions. In more detail, the effects of MLT on TGFbeta secretion would depend on its dosage, as well as on TGF-beta concentrations themselves. MLT could inhibit TGF-beta secretion in the presence of an enhanced TGF-beta production, such as in the advanced neoplasms, and stimulate TGF-beta production in the presence of low TGF-beta levels, such as in the autoimmune diseases [37]. As far as cannabinoids, the main targets for cannabinoid agonists are represented by macrophages and Th17 lymphocytes, by exerting an inhibitory action on IL-17 release from Th17 cells, and on TNF-alpha release from macrophages [29].

The main endogenous cannabinoids are represented by arachidonyl-ethanol-amide (AEA) and 2-arachidonyl-glycerol (2$\mathrm{AG})$, which are mainly produced during the night and during the day, respectively [29]. On the other hand, the two most important, or at least the most studied products of Cannabis are delta-9 tetrahydrocannabinol (THC) and cannabidiol (CBD). THC is the exogenous equivalent of $\mathrm{AEA}$ and 2-AG, while CBD is corresponding to the endogenous palmitoyl-ethanol-amide (PEA), since AEA and 2-AG, as well as THC, exert their activity by directly acting on CB receptors, while CBD and PEA, which are not cannabinoid agonists, may play an indirect action by inhibiting the activity of the fatty acid amide hydrolase (FAAH) [38], the main enzyme responsible for cannabinoid degradation, with a consequent enhanced cannabinoid content. Concerning opioid agonists, the immunosuppressive role of opioids is more typical for the mu-opioid agonists, including morphine and beta-endorphin. On the contrary, the kappa-opioid agonists, including dynorphins, and particularly the delta-opioid agonists, such as met-enkephalin, exert some immunostimulatory actions. Under certain circumstances, e.g., physical exercise, the mu-opioid agonists play transient stimulatory effects on some immune cells, like NK cells, but their chronic action constantly involves a suppression of the immune function [39]. Stress-related immunosuppression is mainly due to an enhanced activation of brain opioid system [30], as well as to the action of the betaadrenergic agonists adrenaline and norepinephrine [32].

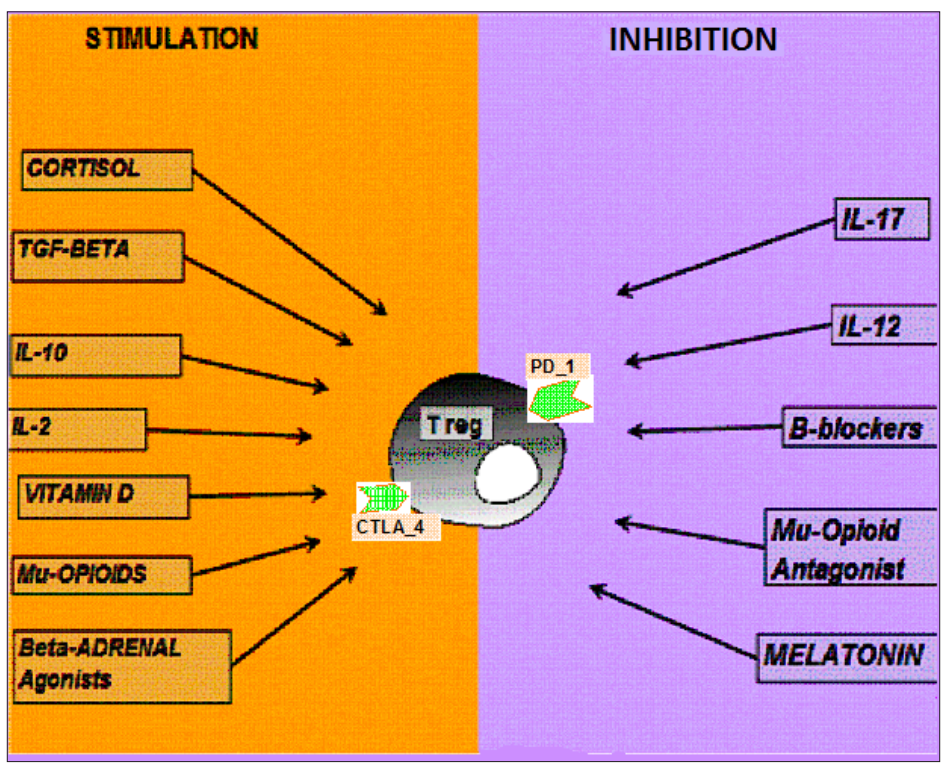

Figure 2: Neuroimmunoregulation of the T-reg lymphocyte. 
Mu-opioid agonists and beta-adrenergic agonists inhibit Th1 cells, cytotoxic T cells, and dendritic cells. On the contrary, T reg cells and Th17 lymphocytes are stimulated by the mu-opioids, at least in some experimental conditions [39]. All three major CD4+ T cells are under a physiological and complex neuroimmunomodulation, the most important being the control of $\mathrm{T}$ reg cell system, because of its regulatory role in the immunoinflammatory processes, and its fundamental immunosuppressive action in anticancer immunity [11]. T reg cells can be identified as CD4+CD25+ Foxp3+ cells, but from a clinical point of view, they may be simply recognized as CD4+CD25+ cells. As illustrated in Figure 2, T reg lymphocytes are stimulated by cortisol, IL-10, TGF-beta, mu-opioid agonists, such as morphine and beta-endorphin, IL-2, vitamin D [40] and beta-adrenergic agonists, in the presence of inhibition of other $\mathrm{T}$ cell subsets. On the contrary, $\mathrm{T}$ reg cells are mainly inhibited by IL-17 [2], and by IL-12 [16], and in a less manner by the other inflammatory cytokines. T reg cells may be also inhibited by betablockers [33], mu-opioid antagonists, including naloxone and naltrexone, and MLT itself, at least in some clinical conditions [35]. As far as cannabinoids are concerned, they would not have direct effects on T reg cells [41], while in vivo they could enhance $\mathrm{T}$ reg cell count by inhibiting IL-17 secretion. It is of particularly interest to consider the activity of beta-blockers, which may play an immunostimulatory action by inhibiting $\mathrm{T}$ reg cell functions, but on the other hand they may also exert an immunosuppressive action when administered at the night, as well as the alpha-2 agonists, because of their inhibitory action on MLT secretion from the pineal gland, which is mainly under a beta-adrenergic regulatory stimulation.

More recently, it has been shown that $\mathrm{T}$ reg cell activation can be counteracted by the administration of anti-checkpoint inhibitor monoclonal antibodies, including the anti-CTLA-4, anti-PD1, and anti PD-L1 and PD-L2 monoclonal antibodies [42]. By summarizing, while the action of stress is constantly promoting tumor onset and dissemination, because of its inhibitory action on Th1 cells and the stimulatory one on both $\mathrm{T}$ reg and Th17 lymphocytes due to the activation of brain opioid system [30], its influence on the possible development of autoimmune diseases would depend on the preferential stimulatory effects of the opioid system on Th17 cells, with a following predisposition to the occurrence of autoimmune processes, or on $\mathrm{T}$ reg cells, which in contrast could absolutely protect against the onset of autoimmune pathologies. The role of $\mathrm{T}$ reg cells in heart pathologies, mainly in the myocardial infarction, as well as in lung inflammatory diseases, is still controversial, since TGF-beta produced by $\mathrm{T}$ reg cells may either reduce the inflammatory response or stimulate the fibrotic processes. In any case, it has been shown that MLT counteracts TGF-beta- induced fibrosis [43].

\section{The Action of Cytokines on the Neuroendocrine System}

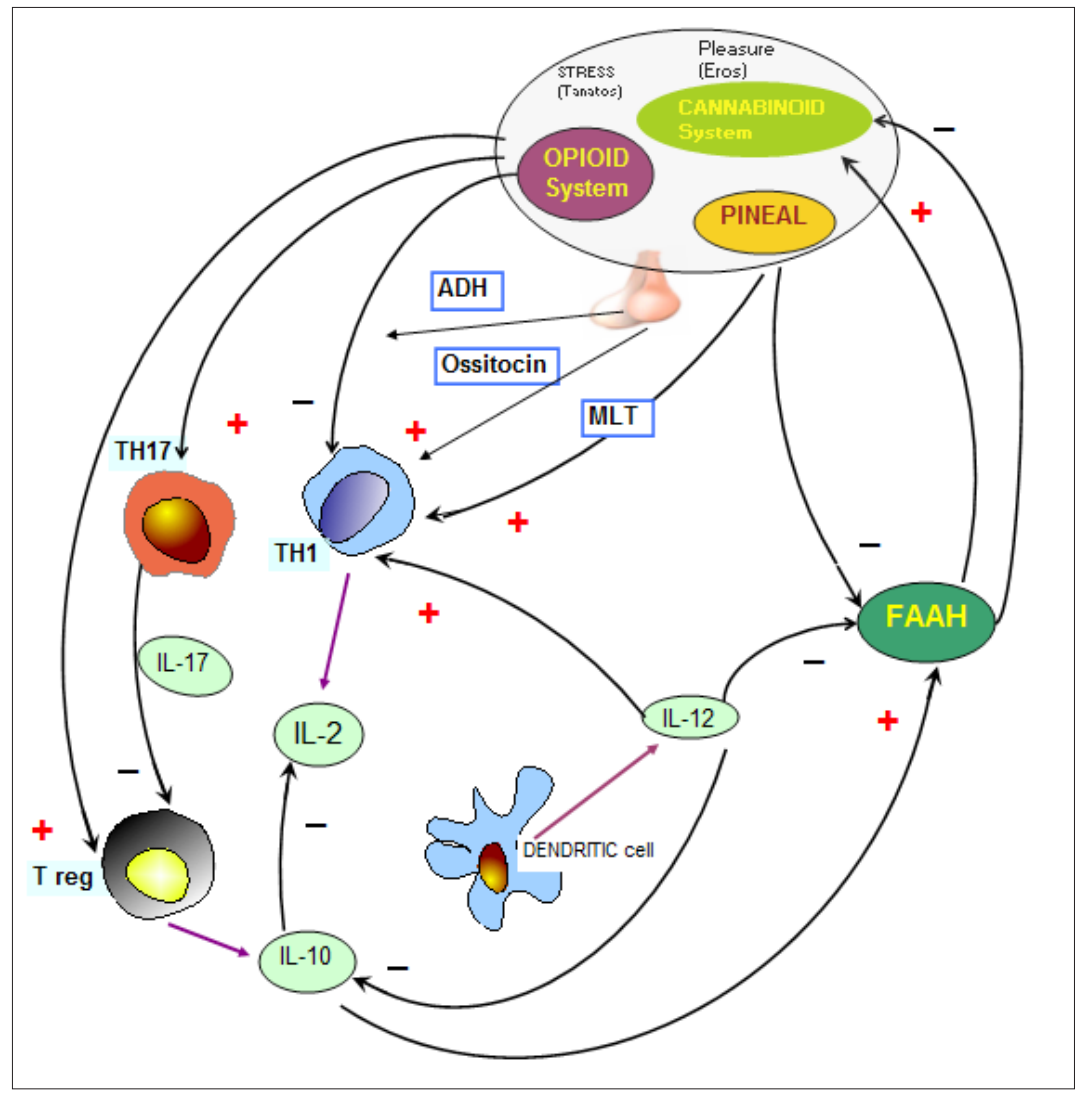

Figure 3: Neuroimmunoregulation of the T-reg lymphocyte. 
According to the foundations of PNEI, the cytokines, whose secretion is under a neuroendocrine modulation, may influence the endocrine and the nervous systems. Then, the immune functions cannot be separated in vivo from the activity of the endocrine and nervous systems. The cytokine regulation of the neuroendocrine system would mainly consisting of the modulation of the activity of FAAH, which is responsible for the functional status of the endogenous cannabinoid system, since an exaggerated activation of FAAH allow an endogenous cannabinoid deficiency, which has been described in most severe clinical conditions, including the disseminated neoplasms and the cardiovascular disorders $[37,44]$. On the other hand, both cannabinoids and MLT can reduce brain and heart ischemic diseases, and prevent the onset of atherosclerosis $[26,29]$. As far as the cytokine regulation of FAAH activity, as illustrated in Figure 3, it has presently been proven that it is mainly determined by IL-12 and IL-10 [45], since IL-12 may inhibit FAAH activity, with a following increase in cannabinoid endogenous content, while IL-10 and IL-4, may stimulate FAAH activity, thus allowing an endogenous cannabinoid deficiency. MLT, cannabinoid agents and CBD all appear to inhibit FAAH activity $[29,37]$. In addition, it has been shown that leptin, a cytokinelike molecule produced by adipocytes, can also stimulate FAAH activity, with a following decline in cannabinoid endogenous content, and this evidence could explain the inhibitory action of leptin on appetite and food intake, which in contrast are enhanced by the cannabinoid agents [46]. The inflammatory cytokines IL-1 beta and IL-6 inhibit in vivo MLT secretion [34], which in contrast is stimulated by the chronic administration of a lowdose of IL-2 [47]. Another fundamental cytokine-neuroendocrine circuit is that represented by the stimulatory effect of most inflammatory cytokines, including IL-1 beta, IL-6, TNF-alpha, IL-2, and interferons, on cortisol secretion, by either inducing CRH and ACTH release at hypothalamic-pituitary site, or directly acting at the adrenal site [48]. The adrenal-inflammatory cytokine circuit would play a fundamental role in preventing the occurrence of autoimmune dynamics during the immune responses against some specific antigens, since cortisol release in response to the action of several inflammatory cytokines may counteract the non-specific proliferation of lymphocytes, including the autoreactive ones, induced by the cytokines produced by the lymphocytes specifically activated against a well-defined antigen, which are more resistant to the suppressive action of cortisol. Then, a reduced cortisol production in response to the inflammatory cytokines during the immune reactions may potentially predispose to the development of autoimmune processes during each specific immune reaction [49].

The Neuroendocrine Modulation of the Inflammatory Response

The most important natural neuroendocrine molecules with anti-inflammatory activity in humans are the cannabinoid agonists [29], and MLT [26] and presumably other pineal indole hormones, such as 5-methoxytryptamine [49], and beta-carbolines, such as the 6-methoxy-1,2,3,4-tetrahydro-beta-carboline, pinealine [50], which have anti-inflammatory, anticancer and psychedelic properties, even though most studies are limited to MLT only. On the contrary, controversial results have been reported for the opioids, which may induce both inflammatory and anti-inflammatory effects. Vitamin D exerts anti-inflammatory effects by stimulating $\mathrm{T}$ reg cell functions and subsequent TGF-beta secretion [41]. Hence it may be effective in the therapy of autoimmune diseases, but it could play a potential pro-tumoral effect, because of the inhibitory action of TGF on the antitumor immunity, even though it may also exert antiproliferative effects. Oxytocin, which plays a fundamental role in the affective life and in the social relationships [31], would also exert remarkable immunostimulatory properties by stimulating Th1 cell activity, even though the clinical studies on the immunomodulatory properties of oxytocin are still at the beginning. The ancient popular evidence of the greater natural resistance to infections and cancer in conditions of pleasure, namely the sexual one, joy and spirituality, may be finally explained according to the PNEI knowledge. In fact, it has been demonstrated that pleasure perception and spiritual sensitivity are chemically mediated by the cannabinoid system, the pineal gland and oxytocin, which have been demonstrated to play antitumoral and immunostimulatory properties.

\section{Age-Related Changes in the Immune Function}

The individual immunoinflammatory biological response depends on several variables, including genetic characteristics, sex, style of life, stress conditions, spiritual sensibility, but the most evident variable is represented by age. Several age-related immune changes have been observed and analysed [51-53], but the most important differences are those concerning the three major T cell subsets. In fact, aged subjects exhibit an increase in Th17 cell activity in association with adiminished Th1 cell function, with the consequent evidence of increased blood levels of IL17 and decreased concentrations of IL-2 [52], and a progressive decline in $\mathrm{T}$ reg cell activity [53]. Therefore, the alterations in immunity occurring with aging may contribute to the development of tumours, severe infective diseases, and autoimmune diseases. Moreover, because of the age-related decrease in circulating MLT levels, the age-related immune changes could depend not only on an altered function of immune cells themselves, but also on the agerelated diminished pineal function [26].

\section{Neuroendocrine Strategies for the Prevention of Cancer and Virus-Induced Complications}

Current knowledge supports the existence of a natural resistance against either cancer onset and growth (which is due to both immune and antitumor antiproliferative actions), or viral infections. A major anticancer endogenous hormone is MLT released from the pineal gland, which also produces other antitumor molecules, such as 5-methoxytryptamine and several beta-carbolines. Even though most oncologists refuse to take into consideration the great number of experimental and clinical studies confirming the fundamental anticancer properties of MLT, 
this compound is the only natural molecule demonstrated to counteract the six main mechanisms responsible for tumor onset and progression $[34,54,55]$, i.e.,

a. abrogation of stress-related predisposition to cancer development

b. prevention of spontaneous or carcinogen-induced malignant transformation

c. inhibition of intercellular junction damage, which is the fundamental condition for the neo-angiogenesis by allowing changes in intercellular matrix composition.

d. inhibition of tumor angiogenesis.

e. inhibition of cancer cell proliferation, through either a stimulation of the anticancer immunity, namely of the IL-2dependent antitumor biological response, or antiproliferative and cytotoxic actions, more evident on tumor expressing MLTreceptor (MT-R), whose prognosis is better than that of MT-R -negative tumours [56].

f. inhibition of FAS-ligand by cancer cells, which is the main mechanism employed by the malignant cells to opposite the action of cytotoxic T lymphocytes.

MLT, as well as 5-methoxytryptamine, at high pharmacological doses have also appeared to protect against DNA damage- and lymphocyte death-induced by x-ray irradiation [57]. MLT is also essential for the maintenance of cannabinoid system function by counteracting an excessive activity of FAAH [58], which could determine an endocannabinoid deficiency, and a consequent predisposition to most human systemic diseases. In fact, abnormally high blood levels of FAAH, and a consequent endocannabinoid system failure, may predispose to cancer and cardiovascular disorders, as well as to more severe complications during viral infections [45]. Then, cannabinoids are another class of essential endogenous molecules responsible for the modulation of the immunobiological response. Since the endogenous cannabinoids, including AEA and 2-AG, are not still available for the treatment of patients, it is justified the use of Cannabis products, THC and CBD. The therapeutic effects of THC and CBD are similar, even though only THC may directly act on CB receptors. Hence, from a clinical point of view, the use of CBD is more suitable than that of THC, since its psychedelic and potentially anxiogenic effects would require a minimal psychotherapeutic assistance. On the contrary, the anxiolytic properties of CBD may furtherly improve the well-being of patients. The most clinically relevant immunomodulatory effect of CBD is given by its stimulatory action on IL-12 [59] in association with an inhibitory effect on IL-10 [59], TNF-alpha and IL-17 [29,37]. By considering that MLT stimulates IL-2 production from Th1 cells and promotes IL-12 secretion from dendritic cells in addition to an inhibitory effect on TNF-alpha and IL-6 production [34], as well as on IL-17 and TGF-beta at least in some clinical conditions [44], the simple non-toxic and non-expensive association of MLT with CBD may already constitute an effective neuroendocrine approach the modulate host immunoinflammatory response. The importance of controlling an eventual excessive host immunoinflammatory reaction, due to an abnormal endogenous secretion of inflammatory cytokines in the absence of an adequate concomitant production of anti-inflammatory cytokines, has dramatically emerged with the planetary disease of Covid-19 infection [23], involving the whole world for the first time of human history.

The abnormal and concomitant endogenous production of the main inflammatory cytokines, including IL-6, TNF-alpha and IL-17, could potentially counteracted by the administration of anti-inflammatory cytokines, such as IL-10, TGF-beta, or specific monoclonal antibodies against the various inflammatory cytokines, such as Tocilizumab for IL-6, Infliximab for TNFalpha and Secukinumab for IL-17, or finally by modulating host immunoinflammatory reactivity through a PNEI approach, by using natural molecules, such as MLT and cannabinoids. In fact, several experimental studies have already shown the ability of MLT to reduce the systemic complications of viral infections [60]. In experimental conditions, the antiviral activity of MLT is due to several mechanisms, including reduction of oxidative stress by acting as free radical scavenger, improvement of the antiviral immune response, and inhibition of the excessive secretion of the inflammatory cytokines [60]. Moreover, MLT has appeared to reverse the immunosuppressive effects of stress in virus infected animals, while there few evidences of a viricidal activity. Then, MLT would act by reducing the severity of viral infections. However, at present there is no relevant study in humans, despite the evident efficacy of MLT in experimental conditions. Obviously, a proposal of a neuroendocrine approach to modulate the biological response requires a well define knowledge of both cytokine systemic effects and systemic disease-related alterations in cytokine secretion and their different immunomodulatory effects. Unfortunately, oldfashion of immunologists does not seem to be able to understand the new medical field of cytokines, since their mind unconsciously tends again to consider the immune reactions only in term of cellcell contact. On the contrary, the knowledge of cytokine functions would require the same mentality of endocrinologists, whose reasoning is in terms of feedback mechanisms. Thus, the main clinical problem is the identification within the great number of altered cytokine secretion which may be those anomalies that provide pathological and prognostic significance. Despite the controversial data reported in the literature, it is already possible to identify the following cytokine secretion anomalies in the main human systemic diseases:

1. Metastatic cancer: progressive decline in IL-2 and IL-12 blood levels in association with abnormally high concentrations of IL-6, IL-1 beta, TNF-alpha, IL-17 and TGF-beta:

2. Acute phase of the autoimmune diseases: abnormally high levels of IL-17, IL-6 and TNF-alpha in association with low levels of TGF-beta and IL-10, while controversial data exist about IL-2 and IL-12 secretions. 
Then, the simple therapeutic neuroimmune regimen with MLT plus CBD may be effective in the treatment of both advanced cancer and autoimmune pathologies by correcting their main cytokine alterations. In more detail, in metastatic cancers, IL-2 deficiency may be corrected mainly by MLT itself, IL-12 deficiency by both MLT and CBD, the high levels of TNF-alpha by both MLT and CBD, and the increased concentrations of TGF-beta and IL-17 by MLT and CBD, respectively. In the acute phase of autoimmunity, the high levels of IL-17 may be controlled by CBD, and those of TNF-alpha and IL-6 by both MLT and CBD. Obviously, a neuroimmune approach in the treatment of human systemic diseases requires a new diagnostic profile, with the proposal of new laboratory examinations, which are generally excluded from the common clinical practices. This new diagnostic approach has at least to include the measurement of the main cytokines, in particular IL-2, IL-6, TNF-alpha, IL-17 and TGF-beta in cancer, IL-17, TGF-beta, IL-2, IL-6 and TNF-alpha in autoimmune pathologies, the evaluation of the circadian light/ dark rhythm of MLT on blood or in an easier manner of the urinary levels of the main MLT metabolite 6-sulfatoxymelatonin, and the investigation of the functional status of the endocannabinoid system, which may be assesses by detecting AEA and 2-AG blood levels, or in simpler manner by measuring the blood levels of FAAH $[29,44]$. The evidence of high levels of FAAH can be considered as the expression of an endogenous cannabinoid deficiency.

\section{Conclusion}

The possibility to modulate and control the individual immunoinflammatory biological response, which is often responsible for the more severe complications of human systemic diseases, represents the future of the medical Sciences. This objective requires a more appropriate knowledge of the physiology of cytokine network, and its influence on all biological systems, as well as of a more complete investigation of the cytokine profile during the clinical course of the systemic pathologies. Unfortunately, the evaluation of cytokine levels in human systemic diseases yet remains an experimental approach, and it is not commonly performed in the clinical management of human diseases. The failure of the clinical evaluation of cytokine secretions during the clinical course of the most important systemic diseases was furtherly worsened by the clinical introduction of the monoclonal antibodies against some specific cytokines, whose secretion has been proven to be abnormally enhanced in some pathologies, in the treatment of human diseases. The dramatic experience of Covid19 infection, whose clinical complications are due to the abnormal endogenous secretion of IL-6, TNF-alpha, and probably in a more important manner of IL-17 [23], can be a unique opportunity to modify this situation.

\section{References}

1. Antony MH (2003) Psychoneuroimmunology and psychoneuroimmunology of cancer: Plausible mechanisms worth pursuing? Brain Behav Immun Suppl 1: 84-91.
2. Korn T Bettelli E, Oukka M, Kuchroo VK (2009) IL-17 and Th17 cells. Annu Rev Immunol 27: 485-517.

3. Grimm EA, Mazumder A, Zhang HZ, Rosenberg SA (1982) Lymphokineactivated killer cell phenomenon. J Exp Med 155(6): 1823-1841.

4. Banks RE, Patel PM Selby PJ (1995) Interleukin-12: A new clinical player in cytokine therapy. Br J Cancer Br J Cancer 71(4): 655-659.

5. Antony PA, Restito NP (2005) CD4CD25+ T regulatory cells, immunotherapy of cancer and interleukin 2. J Immunother 28(2): 120128.

6. Kryczek I, Wei S, Vatan L, Escara-Wilke J, Szeliga W, et al. (2007) Cutting edge: Opposite effects of IL-1 and IL-2 on the regulation of IL-17+ T cell pool. IL-1 subverts IL-2 mediated suppression. J Immunol 179(3): 14231426.

7. Fumagalli L, Lissoni P, De Felice D, Meregalli S, Valsuani G, et al. (1999) Pretreatment serum markers and lymphocyte response to interleukin-2 therapy. Br J Cancer 80(3-4): 407-411.

8. Sakaguchi S, Wing K, Onishi Y, Prieto-Martin P, Yamaguchi T (2009) Regulatory T cells: how they suppress immune responses? Int Immunol 21(10): 1105-1111.

9. Brivio F, Fumagalli L, Parolini D, Messina G, Rovelli F, et al. (2008) T helper/T regulator lymphocyte ratio as a new immunobiological index to quantity the anticancer immune status in cancer patients. In Vivo 22(5): 647-650.

10. Yu Z, Ji M, Yan J, Cai Y, Liu J, et al. (2015) The ratio of Th17/T reg cells as a risk indicator in early acute respiratory distress syndrome. Critical Care 19(1): 82.

11. Zhang B, Rong G, Wei H, Zhang M, Bi J, et al. (2008) The prevalence of Th17 in patients with gastric cancer. Biochem Biophys Res Commun 374(3): 533-537.

12. Zou W (2006) Regulatory T cells, tumor immunity and immunotherapy. Nat Rev Immunol 6(4): 295-307.

13. Shi Y, Wang H, Su Z, Chen J, Xue Y, et al. (2010) Differentiation imbalance of Th1/Th17 in peripheral blood mononuclear cells might contribute to pathogenesis of Hashimoto's thyroiditis. Scand J Immunol 72(3): 250255 .

14. Lissoni P (2017) Therapy implications of the role of interleukin-2 in cancer. Expert Rev Clin Immunol 13(5): 491-498.

15. Chen ZC, Tato CM, Muul L, Laurence A, O'Shea JJ (2007) Distinct regulation of IL-17 in human helper T lymphocytes. Arthritis Rheum 56(9): 2936-2946.

16. King IL, Segal BM (2005) Cutting edge: IL-12 induces CD4+CD25- T cell activation in the presence of T regulatory cells. J Immunol 175(2): 641645 .

17. Langrish CL, Chen Y, Blumenschein WM, Mattson J, Basham B, et al. (2005) IL-23 drives a pathogenic $\mathrm{T}$ cell population that induces autoimmune inflammation. J Exp Med 201(2): 233-240.

18. Lissoni P, Fumagalli L, Rovelli F, Brivio F, Di Felice G, et al. (1998) In vivo stimulation of IL-12 secretion by subcutaneous low-dose IL-2 in metastatic cancer patients. Br J Cancer 77(11): 1957-1960.

19. Whittington R, Faulds D (1993) Interleukin-2. A review of its pharmacological properties and therapeutic use in patients with cancer. Drugs 46(3): 446-483.

20. Cho ML, Jung YO, Moon YM, Min SY, Yoon CH, et al. (2006) Interleukin-18 induces the production of vascular endothelial growth actor (VEGF) in rheumatoid arthritis synovial fibroblast via AP-1-dependent pathways. Immunol Lett 103(2): 159-166. 
21. Dinarello CA (2006) Interleukin 1 and interleukin 18 as mediators of inflammation and the aging process. Am J Clin Nutr 83(2): 447S-455S.

22. Galas A (2014) The determinant of spread of Ebola virus disease - an evidence from the past outbreak experiences. Folia Med Cracov 54(3): 17-25.

23. Li T, Qiu Z, Zhang L, Han Y, He W, et al. (2004) Significant changes of peripheral $\mathrm{T}$ lymphocyte subsets in patients with severe acute respiratory syndrome. J Infect Dis 189(4): 648-651.

24. Paramalingam SS, Thumboo J, Vasoo S, Thio ST, Tse C, et al. (2007) In vivo pro- and anti-inflammatory cytokines in normal and patients with rheumatoid arthritis. Ann Acad Med Singapore 36(2): 96-99.

25. Lissoni P, Barni S, Rovelli F, Tancini G (1991) Lower survival in metastatic cancer patients with reduced inerleukin-2 blood concentrations. Oncology 48(2): 125-127.

26. Brzezinski A (1997) Melatonin in humans. N Engl J Med 336(3): 186195.

27. Di Bella, Scalera G, Rossi MT (1979) Perspectives in pineal function. Prog Brain Res 52: 475-477.

28. Lissoni P, Resentini M, Mauri R, Esposti D, Esposti G, et al. (1986) Effect of tetrahydrocannabinol on melatonin secretion in man. Horm Metab Res 18(1): 77-78.

29. Grotenhermen F (2004) Pharmacology of cannabinoids. Neuroendocrinol Lett 25(1-2): 14-23.

30. Lewis JW, Shavit Y, Terman GV (1983) Apparent involvement of opioid peptides in stress-induced enhancement of tumor growth. Peptides 4(5): 653-658.

31. Khajehei M, Behroozpour E (2018) Endorphins, oxytocin, sexuality, and romantic relationships: an under studied area. World J Obstet Gynecol $7(2): 17-23$.

32. Wahke M, Stachetzki U, Krause A, Pieter M, Hantzschel H, et al. (2001) Regulation of beta2adrenergic receptors on CD4 and CD8 positive lymphocytes by cytokines in vitro. Cytokine 16(6): 205-209.

33. Zhou L, Li Y, Li X, Chen G, Liang H, et al. (2016) Propranolol attenuates surgical stress-induced elevation of the regulatory $\mathrm{T}$ cell responses in patients undergoing radical mastectomy. J Immunol 196(8): 3460-3469.

34. Maestroni GJM (1993) The immunoneuro endocrine role of melatonin. J Pineal Res 14(1): 1-10.

35. Liu H, Xu L, Wei JE, Xie MR, Wang SE, et al. (2011) Role of CD4+CD25+ regulatory $\mathrm{T}$ cells in melatonin-mediated inhibition of murine gastric cancer cell growth in vivo and in vitro. Anat Res (Hoboken) 294(5): 781788.

36. Kuklina EM, Glebezdina NS, Nekrasova IV (2016) Role of melatonin in the regulation of differentiation of $\mathrm{T}$ cells producing interleukin-17 (Th17). Bull Exp Biol Med 160(5): 656-658.

37. Alvarez-Sanchez N, Cruz-Chamorro I, Lopez-Gonzales A, Utrilla JC, Fernandez-Santos JM, et al. (2015) Melatonin controls the experimental autoimmune encephalomyelitis by altering the effector/regulatory balance. Brain BehavImmun 50: 101-114.

38. Nagarkatti P, Pandey R, Rieder SA, Hegde VL, Magarkatti M (2009) Cannabinoids as novel anti-inflammatory drugs. Future Med Chem 1(7): 1333-1349.

39. Gong L, Dong C, Ouyang W, Qin Q (2013) Regulatory T cells: A possible promising approach to cancer recurrence induced by morphine. Med Hypotheses 80(3): 308-310.

40. Lissoni P, Tintori A, Fumagalli L, Bivio F, Messina G, et al. (2008) The endocannabinoid anandamide neither impairs in vitro $\mathrm{T}$ cell function nor induces regulatory cell generation. Anticancer Res 28(6A): 37433748.

41. Aranow C (2011) Vitamin D and the immune system. J Invest Med 59(6): 881-886.

42. Chen J, Jiang CC, Jin L, Zng XD (2016) Regulation of PD-L1: A novel role of pro-survival signaling in cancer. Ann Oncol 27(3): 409-416.

43. Wang YR, Hong RT, Xie YY, Xu JM (2018) Melatonin ameliorates liver fibrosis induced by carbon tetrachloride in rats via inhibiting TGFbeta1/Smadsignaling pathway. Curr Med Sci 38(2): 236-244.

44. Russo EB (2004) Clinical endocannabinoid deficiency (CECD): Can this concept explain therapeutic benefits of cannabis in migraine, fibromyalgia, irritable bowel syndrome and other treatment-resistant conditions? Neuroendocrinol Lett 25(1-2): 31-39.

45. Maccarrone M, Valensise H, Bari M, Lazzarin N, Romanini C, et al. (2001) Progesterone up-regulates anandamide hydrolase in human lymphocytes: role of cytokines and implications for fertility. J Immunol 166(12): 7183-7189.

46. Maccarrone M, Di Rienzo M, Finazzi-Agro A, Rossi A (2003) Leptin activates the anandamide hydrolase promoter in human T lymphocytes through STAT $_{3}$. J BiolChem 278(15): 13318-13324.

47. Viviani S, Bidoli P, Spinazzé S, Rovelli F, Lissoni P (1992) Normalization of the light/ (dark rhythm of melatonin after prolonged subcutaneous administration of interleukin-2 in advanced small cell lung cancer patients. J Pineal Res 12(3): 114-117.

48. Lissoni P, Messina G, Brivio F, Rovelli F, Di Fede G, et al. (2005) Cortisol response to an acute injection of IL-2 in healthy subjects and cancer patients: a first immunoneuroendocrine standardized clinical test to explore the interactions between immune and neuroendocrine systems. J Biol Regul Homeostat Agents 19(3-4): 141-144.

49. Sze SF, Ng TB, Liu WK (1993) Antiproliferative effect of pineal indoles on cultured tumor cell lines. J Pineal Res 14(1): 27-33.

50. Airaksinen MM, Kari I (1981) Beta-carbolines, psychoactive compounds in the mammalian body. Med Biol 59(1): 190-211.

51. Dejaco C, Duftner C, Schirmer M (2006) Are regulatory T cells linked with aging? Exp Gerontol 41(4): 339-345.

52. Hwang KA, Kim HR, Kang I (2009) Aging and human CD4 (+) regulatory T cells. Mech Ageing Dev 130(8): 509-517.

53. Tesar BM, Du W, Shirali AC, Walker WE, Shen H, et al. (2009) Aging augments IL-17 T cell alloimmune responses. Am J Transplant 9(1): 5463.

54. Hill SM, Frasch T, Xiang S, Dupleiss T, Mao L (2009) Molecular mechanisms of melatonin anticancer effects. Integr Cancer Ther 8(4): 337-346.

55. Lissoni P, Rovelli F, Brivio F, Messina G, Lissoni A, et al. (2018) Five yearsurvival with high-dose melatonin and other antitumor pineal hormones in advanced cancer patents eligible for the only palliative therapy. Res J Oncol 2: 1-7.

56. Danielczvk K, Dziegiel P (2009) MT1 melatonin receptors and their role in the oncostatic action of melatonin. Postepy Hig Med Dosw 63: 425434 .

57. Shirazi A, Ghobadi G, Ghazi-Khansari M (2007) A radiobiological review on melatonin: A novel radioprotector. J Radiat Res 48(4): 263-272.

58. Spadoni G, Bedini A, Furiassi L, Mari M, Mor M, et al. (2018) Identification of bivalent ligands with melatonin receptor agonist and fatty acid amide hydrolase (FAAH) inhibitory activity that exhibit ocular hypotensive effect in the rabbit. J Med Chem 17: 7902-7916. 
59. SacerdoteP,Martucci C, Vaccani A, Bariselli F, Panerai AE, Colombo A, et al. (2005) The nonpsychoactive component of marijuana cannabidiol modulates chemotaxis and IL-10 and IL-12 production by murine macrophages both in vivo and in vitro. J Neuroimmunol 159(1-2): 97105.
60. Reiter RJ, Qiang M, Ramaswami S (2020) Treatment of Ebola and other infectious diseases: melatonin "goes viral". Melatonin Res 3(1): 43-57. 